Short Communication

\title{
Thermodynamic properties of DNA-dendrimer complexes and features of their applications
}

\author{
Tamaz Mdzinarashvili ${ }^{1,2}$, Mariam Khvedelidze ${ }^{1,2}$, Eka Shekiladze $^{1,2}$, Nino Shengelia ${ }^{1,2}$ and \\ Tibor Hianik ${ }^{3}$ \\ ${ }^{1}$ Faculty of Exact and Natural Sciences, I. Javakhishvili Tbilisi State University, 3 Chavchavadze ave, 0128, Tbilisi, \\ Georgia \\ ${ }^{2}$ Institute of Medical and Applied Biophysics, I. Javakhishvili Tbilisi State University, 3 Chavchavadze ave, 0128, Tbilisi, \\ Georgia \\ ${ }^{3}$ Faculty of Mathematics, Physics and Informatics, Comenius University in Bratislava, Mlynska dolina F1, 84248 Brati- \\ slava, Slovakia
}

\begin{abstract}
The commercial solution of fourth generation (G4) of poly-amidoamine (PAMAM) dendrimers contains methanol, which is toxic for human body. Our differential scanning calorimetry (DSC) study of dendrimers confirmed the existence of this threat. The recommendation is done on how to prepare dendrimer solutions for practical and safely use in gene delivery. DSC have been also used to study the thermodynamic properties of DNA/dendrimer complexes (dendriplexes). We showed that up to DNA/dendrimer ratio $43 \pm 3(\mathrm{w} / \mathrm{w})$ the solution was homogeneous, but stable aggregates were formed at higher PAMAM content. DSC experiments performed with homogeneous solution of dendriplexes revealed existence of the $\mathrm{pH}$-dependent melting curves that contain several endothermic peaks associated with melting of GC-rich regions.
\end{abstract}

Key words: PAMAM dendrimers — Methanol — DNA — Gene delivery — Differential scanning calorimetry

Dendrimers are highly branched polymer nanoparticles that are considered for decades as possible carriers for drug delivery (Abedi-Gaballu et al. 2018). The intrinsic diversity of dendrimers allows incorporation of various molecules inside their structure or adsorption on dendrimer surface. The evidence exists of incorporation of drugs into the hydrophobic part of dendrimers where they are stabilized by hydrophobic interactions. Hydrophilic molecules can be adsorbed at dendrimer surface containing polar groups. It is known that poly-amidoamine (PAMAM) dendrimers can also interact with short peptides or proteins by electrostatic

Correspondence to: Tamaz Mdzinarashvili, Faculty of Exact and Natural Sciences, I. Javakhishvili Tbilisi State University, 3 Chavchavadze ave, 0128, Tbilisi, Georgia

E-mail: tamaz.mdzinarashvili@tsu.ge

Tibor Hianik, Faculty of Mathematics, Physics and Informatics, Comenius University in Bratislava, Mlynska dolina F1, 84248 Bratislava, Slovakia

E-mail: tibor.hianik@fmph.uniba.sk and hydrophobic forces (Gupta et al. 2006; Melikishvili et al. 2016). In recent years several studies were focused on the application of dendrimers in a gene delivery (Peng et al. 2010; Wang et al. 2011; Jin et al. 2014; Abedi-Gaballu et al. 2018). The gene of interest is transported by nanoparticles into the damaged cell where it can treat multiple genetic diseases. However, there are certain conditions that need to be considered in gene therapy. Among them the safety of the so-called gene vector is crucial. PAMAM dendrimers can be considered as suitable candidates for this purpose. Negatively charged DNA can strongly interact by electrostatic forces with the surface amino groups of PAMAM dendrimers. Such complexes can be obtained with a simple mixing of certain amount of DNA with dendrimers (Peng et al. 2010; Yu and Larson 2014). It should be also pointed out that aggregation of dendrimers in solution is protected due to their excess positive charge. In addition, positively charged dendrimers can interact with the receptors of cell membranes, which can facilitate their penetration into the cells. It is known, that PAMAM dendrimers are positively 
charged at neutral $\mathrm{pH} 7$ and below this value. It should also be taken into account that diameter of G4 generation PAMAM dendrimers is $4.5 \mathrm{~nm}$ (Peng et al. 2010; Yu and Larson 2014). At the same time, the persistent length of double stranded DNA is approximately $50 \mathrm{~nm}$ (Manning 2006). Therefore, dendrimers are in an interior of a coiled DNA, forming maximum interactions with DNA strands. Following to the literature data, after interaction of such complexes with the cell membrane, the endocytosis facilitates the transfection of DNA inside the cell (Kitchens et al. 2007; Peng et al. 2010; Jin et al. 2014). When dendriplexes are transported from the medium with $\mathrm{pH} 7.4$ into the cell with $\mathrm{pH} 5.5$, their surface charge changes in such way that the DNA releases from the nanoparticles and enters the cytoplasm (Peng et al. 2010; Wang et al. 2011). It was established that the DNA transfection performed by dendrimers is several times more efficient than those by other commercial cationic nanoparticles (Peng et al. 2010; Wang et al. 2011). At the same time, the cell enzymatic systems are destroying the nanoparticles (Navath et al. 2008; Jin et al. 2014).

The aim of this work is determination of the thermodynamic properties of commercial PAMAM dendrimers of generation $4(\mathrm{G} 4)$ and the DNA-dendrimer complexes. We also determined the amount of DNA which allow to form a homogeneous complex with these dendrimers. PAMAM G4 dendrimers from Sigma-Aldrich (Germany) diluted in $10 \%$ methanol solution were used. The density of suspension was $0.813 \mathrm{~g} / \mathrm{ml}$, while the molecular weight is $14.2 \mathrm{kD}$. The methanol that is present in commercial dendrimer solution can strongly interacts with both the hydrophobic and hydrophilic part of the dendrimer. We therefore assume that dendrimer interior contains methanol molecules. For calorimetric experiments, the initial concentration of dendrimers was diluted approximately

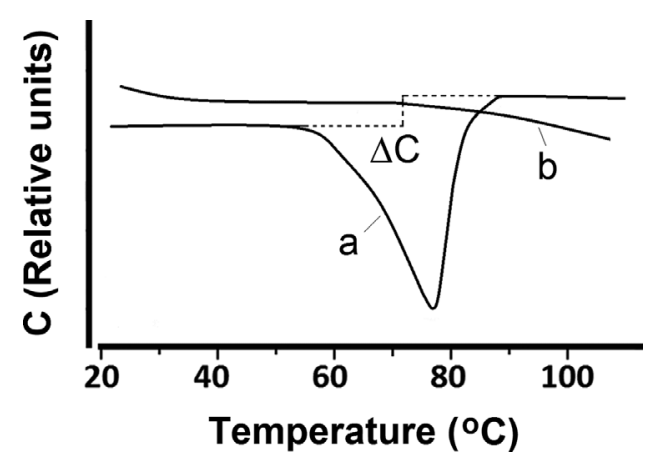

Figure 1A. DSC thermograms of the heat capacity (C) induced by mixing of methanol in water by heating. a) First scan: methanol is in the structure of dendrimer suspension; b) second scan: reheated dendrimers suspension after expected evaporation of methanol and/or by vacuum processing during $1 \mathrm{~min}$. Concentration of dendrimers was $1 \mathrm{mg} / \mathrm{ml}$.
100 times in deionized water at low ionic strength of $15 \mathrm{mM}$ sodium citrate buffer ( $\mathrm{pH} 7$ and/or $\mathrm{pH}$ 3.6). The $\mathrm{pH}$ of the samples was determined directly before experiments in the Ependorf tube using a micro $\mathrm{pH}$ meter from Hanna Instruments (Italy). Calorimetric experiments were performed using the differential adiabatic microcalorimeter DASM-4A (Russia) with working volume of $0.54 \mathrm{ml}$. From the calorimeter we get the data in a digital form about the temperature and the difference heat capacity for samples, which were then processed by graphic programs. The exact $\Delta \mathrm{C}=\mathrm{dQ} / \mathrm{dt}$ values were then determined using the standard sample. We used DNA from calf thymus composed of 41.9 mole \% G-C and 58.1 mole \% A-T (Sigma-Aldrich, USA). The concentration of DNA used in experiments ( $c=1.5$ $\mathrm{mg} / \mathrm{ml}$ ) was determined by spectrophotometer UPS 3800 (Thermo Fisher Scientific, USA).

Before determination of the thermodynamic properties of DNA-dendrimer complexes we performed calorimetric study of pure dendrimers. For this purpose, the dendrimers were diluted with deionized water for final concentration of $1 \mathrm{mg} / \mathrm{ml}$. Figure 1 (curve a) shows the changes in heat capacity (C) as a function of temperature. It can be seen clear exothermic effect at $80^{\circ} \mathrm{C}$. After cooling the sample in the calorimeter to the room temperature and measuring the calorimetric curve again (i.e. the reheating experiment) the exothermal heat effect was no longer observed (Fig. 1, curve b). This can be explained by irreversible leaking of the methanol out of the dendrimer interior with subsequent evaporation from the sample. This assumption is confirmed by literature data on which G4 PAMAM dendrimers are much better soluble in methanol than in water (Peterson et al. 2001). However, after heating the sample the methanol moves from the dendrimer structure into the water. This happens due to the boiling methanol inside the dendrimer (the temperature of methanol boiling is $64^{\circ} \mathrm{C}$ ). This boiling causes disturbance of dendrimer structure and mixing with the water. This process must be accompanied by heat emissions (Peeters and Huyskens 1993), which we have observed in calorimetric experiments. This assumption is confirmed by the beginning of the exothermic process at the $60^{\circ} \mathrm{C}$ region shown on Fig. 1, curve a. At the same time, this exothermic effect is not present at the second scan measured after sample heating, because the methanol evaporation is irreversible. Thus, heating the sample above $80^{\circ} \mathrm{C}$ resulted in removal of the methanol from dendrimer interior. Based on comparison of the area of the calibrated and the thermal peak (Fig. 1, curve a) we estimated exothermal energy corresponding to the methanol removal from the dendrimers: $29 \mathrm{~mJ}$. The known value of the specific heat of dissolution of methanol in water $(270 \mathrm{~kJ} / \mathrm{kg}$, see Peeters and Huyskens 1993) allows calculation of the amount of methanol, which is mixed with water. We can calculate the mass (M) of methanol, which can cause an exothermic ef- 
fect equal to $\Delta \mathrm{Q}=29 \mathrm{~mJ}: \mathrm{M}=29 \times 10^{-3} / 270 \times 10^{3}=0.11 \mathrm{mg}$ of methanol. This mass of methanol molecules is presented in $0.54 \mathrm{mg}$ of dendrimer solution (working volume of the calorimeter is $0.54 \mathrm{ml}$ and dendrimer concentration was $1 \mathrm{mg} / \mathrm{ml}$ ). Thus, we assume that most of the methanol molecules in the untreated dendrimer solution are in the dendrimer interior. This conclusion is based also on the peculiarities of the partitioning of amphiphilic molecules (methanol) in water-dendrimer phase that accelerate the incorporation of methanol into the dendrimers (Manning 2006). However, after the heating of dendrimer solution the methanol is replaced by water molecules. This is confirmed by existence of a change of heat capacity $(\Delta C)$, shown in Figure 1. The above-mentioned assumption has been confirmed also by samples vacuum processing (sample was placed in a vacuum for one minute) prior staring the calorimetric measurements. This procedure resulted in methanol release from dendrimer structure and calorimetric curve did not show any exothermal effects (Fig. 1, curve b). Scan presented by curve b was identical for dendrimers processed by heating and those after vacuum processing during $1 \mathrm{~min}$.

The obtained result suggests that the toxicity of dendrimers may be particularly due to the presence of methanol in non-preheated dendrimers in addition to the positive dendrimer surface charge. Thus, without preheating the methanol from dendrimers can be released from dendrimers after uptake into the cell and cause the toxicity. We believe that the obtained results are important if the commercial G4 PAMAM dendrimers are used as drug carriers. Thus, prior medical application the dendrimers should be processed in advance by vacuum or by temperature heating.

We also studied the thermodynamic properties of the complexes of DNA with methanol-free G4 PAMAM dendrimers (dendriplexes). Dendrimers have been treated under the vacuum before mixing with DNA for metha- nol removal. For obtaining dendriplexes it is necessary to fulfill certain conditions. First, at neutral and acidic conditions the phosphate groups of DNA are negatively charged and dendrimers are positively charged. Second, certain DNA/dendrimer ratio should be selected for formation of proper complexes to provide homogeneous dendriplex solution.

The calorimetric experiments were carried out at different $\mathrm{pH}$ and at two different weight ratios of DNA/ dendrimer. The DNA/dendrimer complexes were formed as follows. A DNA solution with a concentration $1.5 \mathrm{mg} /$ $\mathrm{ml}$ was titrated with small portions $(0.033 \mathrm{ml})$ of the dendrimer solution in a concentration of $0.258 \mathrm{mg} / \mathrm{ml}$. We have found that the solution remained homogeneous up to DNA/dendrimer ratio $43 \pm 3(\mathrm{w} / \mathrm{w})$. Addition of the next portion of the dendrimers resulted in formation of visible inhomogeneities. These aggregates were practically irreversible, since it was impossible to disassemble them by sonication. For calorimetric experiments we used only homogeneous solutions of DNA/denrimer complexes 43 and $65.8(\mathrm{w} / \mathrm{w})$. Figure 2 shows the temperature scan of specific heat capacity $\left(C_{p}\right)$ for pure DNA (concentration $1.5 \mathrm{mg} / \mathrm{ml}$ ) and for dendriplexes at above mentioned two DNA/dendrimer weight ratios at $\mathrm{pH} 7$ (A) and $\mathrm{pH} 3.6$ (B). At neutral and acid $\mathrm{pH}$ the typical melting process was observed for pure DNA (Fig. 2A, curve a). The effect of $\mathrm{pH}$ on the conformation of polynucleotide chains in solution is due to the fact that hydrogen bonds stabilizing the helical structure of DNA are formed in these molecules between the nitrogen bases of the single strands. These nitrogen bases are capable of ionization in an acid medium, so the ionization of at least one of the base participating in the hydrogen bond between the DNA strands means the rupture of the latter (breaking the hydrogen bond). This resulted in a change of stability of the DNA molecule. A dissociation constant of nitrogen groups is in the range of $\mathrm{pH}$
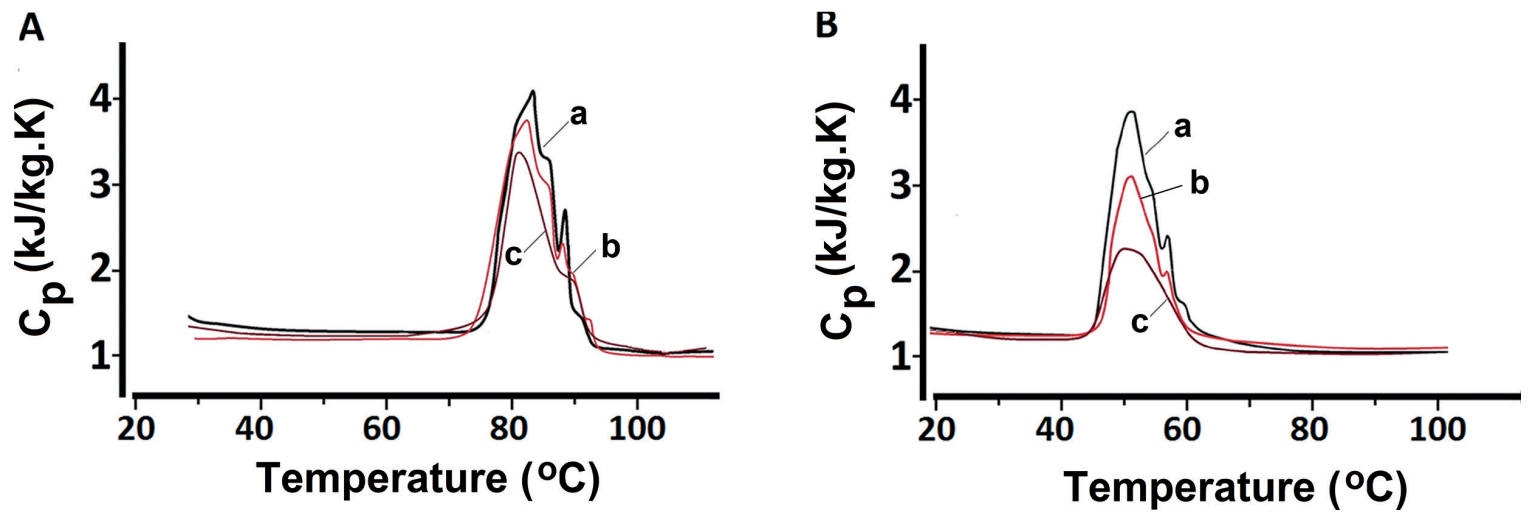

Figure 2. Temperature scans of the specific heat capacity $\left(C_{p}\right)$ for pure DNA (a) and for DNA/dendrimer ratios 43 (w/w) (b) and 65.8 $(\mathrm{w} / \mathrm{w})(\mathrm{c})$ at different $\mathrm{pH}$ : $\mathrm{pH} 7(\mathbf{A})$ and $\mathrm{pH} 3.6(\mathbf{B})$. The concentration of DNA was $1.5 \mathrm{mg} / \mathrm{ml}$. 


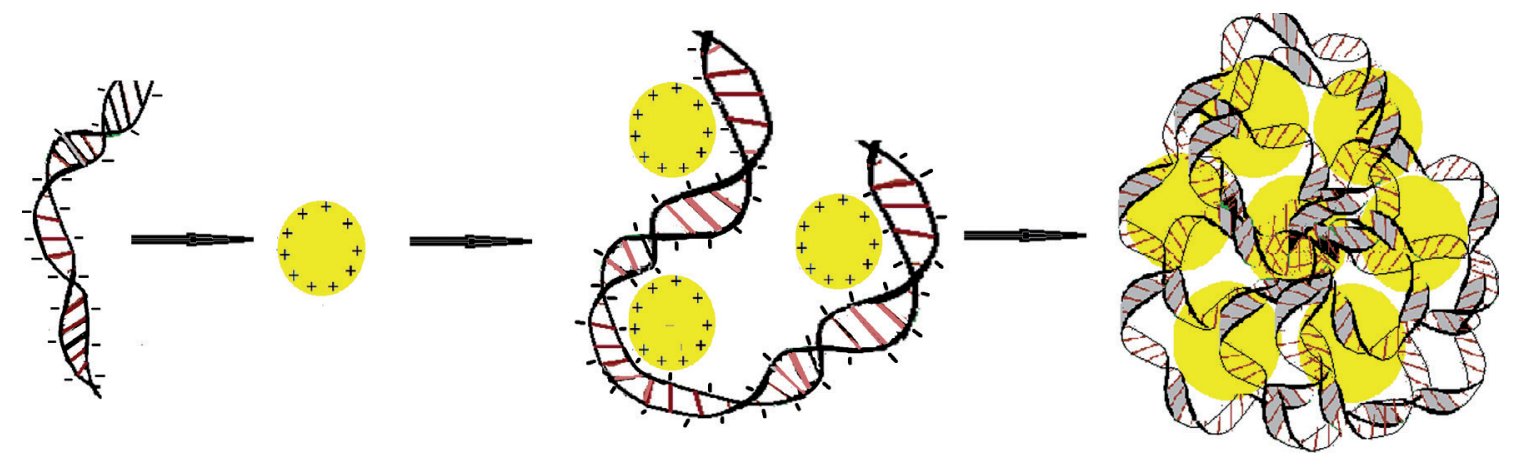

Figure 3. The scheme of formation the complex between DNA and G4 generation PAMAM dendrimers.

3-5 (2.9 (guanine); 3.7-3.8 (adenine); 4.5-4.8 (cytosine)). According to these data at an acidic environment ( $\mathrm{pH} 3.6)$ the hydrogen bonds are weakened, and therefore the DNA denaturation temperature decreases (from $82^{\circ} \mathrm{C}$ to $52^{\circ} \mathrm{C}$ ). The shape of the denaturation peak also changed. This agree well with our previous publication (Mdzinarashvili et al. 2009).

The fact, that complexes of DNA-dendrimer were formed, follows from the calorimetric curves. The DNA-dendrimer complexes are characterized by different shape and enthalpy (area under the peak) in comparison with those of pure DNA. In addition, the calorimetric curves depend on the DNA/dendrimer ratio (Figures $2 \mathrm{~A}$ and $\mathrm{B}$ ). The melting curve contains several endothermic peaks that are associated with melting of GC-rich regions (with increasing of DNA/ dendrimer ratio, the high temperature peaks disappear). This is typical thermodynamic behavior for so-called "fine structure" stabilized by hydrogen bonds of various strength (AT base pairs are weaker than GC pairs). At presence of dendrimers the main melting peak as well as GC peaks are substantially reduced. This may suggest preferable interaction of dendrimers with GC-rich regions of DNA. At the acidic $\mathrm{pH}$ ( $\mathrm{pH}$ 3.6) the melting curve of pure DNA is similar to those at neutral $\mathrm{pH}$. However, the high temperature peaks are smaller which is evidence of certain destabilizing effect of acidic conditions on the double helix. The acidic $\mathrm{pH}$ more strongly reduced main melting peaks as well as GC melting peaks. We should also note that the DNA-dendrimer complexes are formed between the positively charged surface of the dendrimers and negatively charged phosphate groups of DNA (Wong et al. 2014). Therefore, the change of $\mathrm{pH}$ from neutral to acidic ( $\mathrm{pH} \mathrm{3-7)} \mathrm{can} \mathrm{not} \mathrm{significantly} \mathrm{change} \mathrm{the}$ size of the dendrimer (maximal changes (swells) can be around 5\%, see Maiti et al. 2005) as well as the charge of the DNA and dendrimers. Consequently, protonation does not affect substantially the formation of the complexes. Recalculating of weight ratio to molar ratio one can obtain $923 \pm 65$ base pairs of DNA per one dendrimer molecule. The scheme of formation DNA-dendrimer complexes is presented on Figure 3. Please note, that this picture is not in a scale.

Our results demonstrate that for safe application of PAMAM dendrimers as a gene delivery system it is necessary to pretreat commercially available dendrimers that are typically dissolved in methanol using either heating up to minimum $80^{\circ} \mathrm{C}$ or processing in vacuum to remove toxic methanol from the dendrimer interior. Calorimetric study of DNA-dendrimer complexes indicates that at presence of dendrimers the main as well as high temperature DNA melting peaks decrease, which is evident of strong binding of DNA with dendrimers.

Acknowledgments. This work was financially supported by Science Grant Agency VEGA (project No. 1/0152/15) and by Agency for Promotion Research and Development under the contract APVV-14-0267. This contribution is also the result of the project implementation „Development of the Centre of Excellence for Exploitation of Informational Biomacromolecules for Improvement of Quality of Life" project supported by the Research and Development Operational Programe funded by the ERDF (Contract No. ITMS 26240120027).

\section{References}

Abedi-Gaballu F, Dehghan G, Ghaffari M, Yekta R, AbbaspourRavasjani S, Baradaran B, Ezzati Nazhad Dolatabadi J, Hamblin MR (2018): PAMAM dendrimers as efficient drug and gene delivery nanosystems for cancer therapy. Appl. Mat. Today 12, $177-190$ https://doi.org/10.1016/j.apmt.2018.05.002

Gupta U, Agashe HB, Asthana A, Jain NK (2006): Dendrimers: novel polymeric nanoarchitectures for solubility enhancement. Biomacromolecules 7, 649-658 https://doi.org/10.1021/bm050802s

Jin L, Zeng X, Liu M, Deng Y, He N (2014): Current progress in gene delivery technology based on chemical methods and nano-carriers. Theranostics 4, 240-255 https://doi.org/10.7150/thno.6914 
Kitchens KM, Foraker AB, Kolhatkar RB, Swaan PW, Ghandehari $\mathrm{H}$ (2007): Endocytosis and interaction of poly (amidoamine) dendrimers with Caco-2 cells. Pharm. Res. 11, 2138-2215 https://doi.org/10.1007/s11095-007-9415-0

Manning GS (2006): The persistence length of DNA is reached from the persistence length of its null isomer through an internal electrostatic stretching force. Biophys.J. 91, 3607-3616 https://doi.org/10.1529/biophysj.106.089029

Maiti PK, Çagin T, Lin S-T, Goddard WA (2005): Effect of solvent and $\mathrm{pH}$ on the structure of PAMAM dendrimers. Macromolecules 38, 979-991 https://doi.org/10.1021/ma0491681

Mdzinarashvili T, Partskhaladze T, Khvedelidze M, Lomidze T (2009): Consequences of an acidic environment for the structural and functional abilities of DNA. J. Biol. Phys. Chem. 9, 77-82

Melikishvili S, Poturnayova A, Ionov M, Bryszewska M, Vary T, Cirak J, Mu-oz-Fernández MA, Gomez-Ramirez R, de la Mata FJ, Hianik T (2016): The effect of polyethylene glycol-modified lipids on the interaction of HIV-1 derived peptide-dendrimer complexes with lipid membranes. Biochim. Biophys. Acta 1858, 3005-3016 https://doi.org/10.1016/j.bbamem.2016.09.005

Navath RS, Kurtoglu YE, Wang B, Kannan S, Romero R, Kannan RM (2008): Dendrimers-drug conjugates for tailored intracellular drug release based on glutathione levels. Bioconjug. Chem. 12, 2446-2455 https://doi.org/10.1021/bc800342d

Peeters D, Huyskens P (1993): Endothermicity or exothermicity of water/alcohol mixtures. J. Mol. Structure 300, 539-550 https://doi.org/10.1016/0022-2860(93)87046-C

Peng SF, Su CJ, Wei MC, Chen CY, Liao ZX, Lee PW, Chen HL, Sung HW (2010): Effects of the nanostructure of dendrimer/ DNA complexes on their endocytosis and gene expression. Biomaterials 21, 5660-5670 https://doi.org/10.1016/j.biomaterials.2010.03.059

Peterson J, Allikmaa V, Penko T, Lopp M (2001): Fragmentation of PAMAM dendrimers in methanol. Proc. Estonian Acad. Sci. Chem. 50, 167-172

Wang H, Shi H, Yin SK (2011): Polyamidoamine dendrimers as gene delivery carriers in the inner ear: How to improve transfection efficiency. 5, 777-781 https://doi.org/10.3892/etm.2011.296

Wong PT, Tang K, Coulter A, Tang S, Baker JR, Choi SK (2014): Multivalent dendrimer vectors with DNA intercalation motifs for gene delivery. Biomacromolecules 15, 4134-4145 https://doi.org/10.1021/bm501169s

Yu S, Larson RG (2014): Monte-Carlo simulations of PAMAM dendrimer-DNA interactions. Soft Matter 9, 5325-5336 https://doi.org/10.1039/C4SM00452C

Received: April 9, 2018

Final version accepted: August 20, 2018 